\title{
el control de calidad de los hormigones a través de los ensayos de probetas a compresión simple
}

\author{
JOSE M. LOPEZ SAIZ, Ingeniero de Caminos, Canales y Puertos - A. M. A.S.C.E
}

\begin{abstract}
simopsis
En el presente artículo se exponen algunas ideas acerca del control estadístico de la calidad de los hormigones empleados en las obras. Se comentan las tendencias actuales de dicho control y se aclaran, mediante un ejemplo tomado de una obra real, el puente de Navacerrada en el Nudo de Villalba, ejecutado por Huarte y Cía., S. A., con proyecto de D. Carlos Fernández Casado, sobre el cual su autor informó ampliamente en un artículo aparecido en esta misma revista.
\end{abstract}

La función primordial perseguida por los ensayos de compresión simple es asegurar una producción uniforme del hormigón con una resistencia y calidad especificadas.

Son muchas las variables que se reúnen al fabricar un hormigón y que influyen en sus propiedades.

Las variaciones en las propiedades de un hormigón, por tanto, han de aceptarse como cosa natural aun fabricado en condiciones, a nuestro juicio, exactamente iguales.

No obstante, es necesario saber interpretar debidamente los diversos ensayos y sus variaciones para poder juzgar la suficiente calidad de un hormigón y ser conscientes de las limitaciones de los métodos empleados.

Siempre ha de tenerse en cuenta que para lograr un hormigón de calidad no basta fijarse en la resistencia de las probetas fabricadas con una porción del mismo. En primer lugar, la resistencia no es la única propiedad de un hormigón, ni tan siquiera la más importante en muchas ocasiones. La retracción, la fluencia bajo carga, la durabilidad, resistencia a la helada, resistencia a los agentes exteriores, al desgaste, agentes químicos, resistencia a la tracción, reacciones de corrosión sobre las armaduras, etc., son muchas veces propiedades primordiales.

El control de la resistencia es, sin embargo, de gran importancia, pues se han encontrado relaciones cuantitativas o cualitativas entre la resistencia a compresión y la mayor parte de las restantes propiedades. No obstante, cuando una de estas propiedades sea de gran importancia, habrá que hallar su relación cualitativa con la resistencia a compresión simple del hormigón ejecutado, ya que dicha relación varía con la dosificación, granulometría, etc., empleados.

Al mismo tiempo existe una relación entre la resistencia obtenida de las probetas y la resistencia real del hormigón y que depende de la forma de curado, temperatura a que está sometido el hormigón durante el fraguado, etc.; variables que en obra suelen ser distintos del sistema normalizado de curado que se sigue para las probetas. 
Ensayos recientes (Richard H. Cambel and Robert E. Robin, «Core and Cilinder Strengths». Journal of the A.C.I., abril 1967) han demostrado que en testigos obtenidos, del hormigón puesto en obra, mediante sondas rotativas, la resistencia del hormigón es un $10 \%$ menor que la de testigos curados en obra en idénticas condiciones, debido a la fisuración interna que en el testigo produce la sonda al romper los áridos; de aquí, la gran dificultad de obtener datos concretos de la resistencia de hormigones en obra, al menos siempre que los testigos se perforen entre los 14 y 21 días, momento en que se hizo en los ensayos citados.

Todo esto hace pensar en las grandes dificultades que lleva consigo el apreciar la resistencia de un hormigón puesto en obra. Sin embargo, los estudios de las Delegaciones Suiza, Española y Belga del C.E.B. llegaron a la conclusión de que, para el cálculo de secciones rectangulares, la mejor representación de la resistencia del hormigón se obtiene comparándola con la resistencia a rotura de probetas cilíndricas de $15 \times 30$ curadas en idénticas condiciones.

Las diferencias entre el hormigón puesto en obra y las probetas en trabajos normales, son tenidas en cuenta de una manera práctica dentro de los coeficientes de seguridad empleados en el cálculo de estructuras de hormigón.

Como conclusión podemos decir que el ensayo de probetas cilíndricas de $15 \times 30 \mathrm{~cm}$ a compresión simple es un método poderoso para estimar la calidad de un hormigón, siempre que se interpreten correctamente los resultados y, en general, dan una indicación de la uniformidad de un hormigón detectando variaciones «no estadísticas» y facilitando que se localicen las causas de dichas variaciones.

En la «Instrucción para el proyecto de Obras de hormigón», actualmente vigente, se toma como resistencia a rotura la máxima carga unitaria obtenida a los 28 días en probetas cilíndricas de altura doble del diámetro, afectándose, para el cálculo, esta resistencia, de un coeficiente de seguridad 3. La interpretación lógica de esta norma es obtener la resistencia de un hormigón como media de las resistencias del conjunto de probetas elaborado con ese hormigón. Automáticamente, aparece una duda, y es la de que hormigones muy distintos en su ejecución pueden dar lugar a resistencias medias iguales, siendo sus resistencias parciales muy dispares. La primera solución que se da a este problema, muy simple, y a la vez creadora de problemas mayores, es la de ir haciendo ensayos y obligar a que todos den valores superiores al exigido. Como norma, pues, se ejecutaban series de probetas de una amasada y se exigía que su media fuera superior a la resistencia de cálculo.

Esta exigencia encerraba un concepto de «valor mínimo admisible» que poco a poco se fue señalando como motivo de revisión a la luz de nuevos conceptos estadísticos que hacen ver la posibilidad de que aparezcan valores menores que uno dado, asociados a una probabilidad de aparición mayor a menor.

La Norma H.A. 61 aborda el problema de los ensayos a compresión simple de probetas de hormigón como pertenecientes a un universo estadístico que se distribuye de forma "normal» e intenta introducir un sentido estadístico en el concepto de resistencia característica al definirla como el resultado de disminuir la resistencia media en el valor de la desviación típica de los resultados obtenidos, es decir:

$$
\sigma_{b k}=\sigma_{b m}-S,
$$

en la cual:

$$
\begin{aligned}
\sigma_{b k} & =\text { resistencia característica; } \\
\sigma_{b m} & =\text { resistencia media; } \\
S & =\text { desviación típica de los resultados empleados para obtener } \sigma_{b m} .
\end{aligned}
$$


En esta Norma H.A. 61 todo el concepto de resistencia característica va ligado al de coeficiente de seguridad como viene indicado en el anejo de los comentarios y a la vez al valor de la desviación típica del universo a que pertenecen las probetas ensayadas, no a la desviación típica de los resultados obtenidos; valores que son muy semejantes para un número de ensayos grande, pero que difiere para el número de ensayos pequeño, ya que la desviación típica de la muestra es un estimador de la desviación típica del universo, pero con un sesgo, es decir:

$$
\delta^{2}=\frac{n}{n-1} \cdot S^{2}
$$

siendo:

$\delta=$ desviación típica del universo al que pertenece la muestra.

La resistencia característica se define como aquella que tiene la propiedad de que sólo 1/6 de las probetas del universo estadístico formado por el hormigón estudiado tienen una resistencia menor que la característica. Siguiendo esta definición obtendremos la fórmula:

$$
\sigma_{b k}=\sigma_{b m}-0,967 \delta ;
$$

ecuación muy próxima a la anterior para un número de ensayos normal. Sin embargo, para definir un método práctico de calcular $\sigma_{b k}$ se hacen aproximaciones que llevan a obtener como valor de $\sigma_{b k}$ la media de la mitad de los resultados más bajos del conjunto de ensayos; no obstante, esta aproximación equivale a obtener el valor de la resistencia característica de la fórmula:

$$
\sigma_{b k}=\sigma_{b m}-0,8 \delta .
$$

Como se puede apreciar siguiendo el mismo desarrollo del anejo citado, la comprobación experimental de esta fórmula aproximada con relación a la fórmula [3] ha sido mostrada con motivo de la construcción del Puente de Toncarville (G. Dreux, "Contribution a l'Étude du Comportament des Eprouvettes de Béton». Annales de l'I.T.B.T.P., febrero 1965).

Como se ve de lo expuesto anteriormente, en la Norma H.A. 61 no queda debidamente centrado el concepto de resistencia característica, que se define como aquello que tiene una posibilidad de no ser alcanzado una vez en 6 ensayos y se aproxima por aquélla en que esta probabilidad es de aproximadamente 1:5. Como dice también que para todos los efectos prácticos se puede tomar este último valor, lo haremos así.

Ello nos lleva a una nueva ecuación, que es la que tomaremos como definitiva:

$$
\sigma_{b k}=\sigma_{b m}-0,842 \delta .
$$

Se ha de hacer notar que en esta fórmula $\delta$ es la desviación típica del conjunto total. Si se emplea para calcular $\sigma_{b k}$ la desviación típica de los ensayos empleados, para obtener $\sigma_{b m}$ esta fórmula ha de variarse tal como se indica más adelante.

Desgraciadamente, la interpretación que se ha dado en muchos casos a la forma de aplicar esta resistencia característica no ha sido estadística en absoluto, y se controla el hormigón mediante la obtención de una serie de probetas de las amasadas del hormigón que se van poniendo en obra y se exige que la resistencia característica de cada una de estas series sea igual o superior a la resistencia característica exigida.

Con esta interpretación se da nuevamente lugar a una exigencia de valor «mínimo admisible» a lo que se han de poner las mismas objeciones que anteriormente, con el agravante de que 
ésta es más dura que la anterior, ya que $\sigma_{b k}=\sigma_{b m}-0,8 \delta \mathrm{y}$, por tanto, $\sigma_{b k} ₹ \sigma_{b m}$ en todos los casos.

Pero se hace más patente la falsedad de esta interpretación al pensar que la resistencia obtenida en una serie de probetas de una amasada no nos indica en absoluto las variaciones en el hormigón que se está ejecutando, ya que éstas aparecen de amasada a amasada. La dispersión obtenida en una serie no es más que indicativa de la dispersión del ensayo y, por consiguiente, no se ha de tomar más que como representación de la calidad y el cuidado puesto en el mismo. Esta variación es equivalente a la que se encuentra al medir una dimensión de una pieza, lo cual no quiere decir que esa dimensión varíe en cada medida, sino que estas medidas no son exactas y se distribuyen con una cierta función de distribución alrededor del verdadero valor; por tanto, en una serie de probetas el valor de la dispersión tiene aplicación para el control de la calidad del ensayo, y la resistencia media, representativa de la resistencia de la amasada, es la que se ha de tomar en cuenta para estimar la resistencia del hormigón que se está ejecutando.

Supongamos que hacemos una serie de probetas de una muestra del hormigón que estamos ejecutando. Evidentemente, esta muestra tendrá una resistencia definida y los resultados del ensayo se distribuirán, con una cierta función de distribución, alrededor de este valor. Es normal aceptar que el valor real se puede estimar como la media de un número suficientemente grande de ensayos; ahora bien, supongamos que los resultados se distribuyen según una distribución «normal» con media $\sigma_{m}$ y la desviación típica $\delta$, entonces, siendo $n$ el número de probetas de la serie, la probabilidad de que la media esté contenida en el intervalo

$$
R_{m} \pm \frac{T_{p} \cdot S}{n-1}
$$

es $\quad 1-\frac{p}{100}$

siendo:

$T_{p}=$ valor de $p$ por ciento de la $t$ de Student para $n-1$ grados de libertad;

$S=$ desviación típica obtenida de la muestra;

$R_{m}=$ media de dicha muestra.

Si ahora llamamos $V_{1}$ al coeficiente de variación obtenido a partir de los resultados del ensayo, es decir, al cociente de la desviación típica por la media, el error máximo que se puede cometer con una probabilidad del $\left(1-\frac{p}{100}\right) \%$ es:

$$
E \%= \pm \frac{T_{p} \cdot V_{1}}{\sqrt{n-1}}
$$

Si el número de ensayos efectuados es suficientemente grande y mediante él calculamos la desviación típica debido al ensayo como medida de los distintos ensayos, el valor medio del error será:

$$
E \%= \pm \frac{\lambda_{p} \cdot V}{\sqrt{n}}
$$

en la que $\lambda_{p}=$ valor de la distribución normal para el $p \%$, ya que la media de una muestra de $n$ valores se distribuye de forma normal con media $\sigma_{m}$ y desviación típica $\delta / \sqrt{n}$. 
En este caso, con un coeficiente de variación del $5 \%$, que es el valor máximo para ensayos normales y series de 3 probetas, el error máximo puede ser $E \%=4,8 \%$, con una probabilidad del $90 \%$; es claro, por lo tanto, que bastan series de 3 probetas para obtener una aproximación suficiente de la medida representativa de la resistencia del hormigón del que se obtuvo la serie.

Por supuesto que la resistencia del hormigón de una amasada podemos caracterizarla por su resistencia media o por su resistencia característica; de esta segunda forma añadimos un coeficiente de seguridad función de la dispersión del ensayo, garantizando que existe una probabilidad del $80 \%$ de que el valor de la resistencia de la amasada sea superior al valor tomado. El coeficiente de seguridad, sin embargo, está estudiado con relación a la resistencia media de probetas cilíndricas de $15 \times 30$, cuidadas en idénticas condiciones que el hormigón estructural; por eso, al elegir como resistencia representativa de una muestra la característica de la serie de probetas en lugar de la media, equivale a tomar un coeficiente de seguridad adicional de:

$$
\frac{1}{1-0,84 V}=\frac{1}{0,958}=1,04 \text {; }
$$

suponiendo para $V$ el valor de 0,05 , que es el que se considera como normal en ensayos cuidadosos.

Para obtener la resistencia que represente a una muestra del hormigón en ejecución se ha de estudiar la serie de probetas ejecutadas con dicha muestra para ver si todas ellas pertenecen a la misma población estadística; es decir, si no existe ninguna causa por la cual una probeta haya roto a la carga que la ha hecho, en general se supone que existe una "causa asignable» cuando la probeta rompe a valores distanciados del valor medio $\pm 3 \delta$, suponiendo para ello conocido el valor de $\delta$ para el ensayo en cuestión o estimándolo a partir de la resistencia de las probetas de la serie ejecutada mediante la fórmula:

$$
\delta=\sqrt{\frac{n}{n-1}} \cdot \sqrt{\frac{\sum\left(R_{b i}-R_{b m}\right)^{2}}{n}}=\sqrt{\frac{\sum\left(R_{b i}-R_{b m}\right)^{2}}{n-1}}
$$

siendo:

$$
\begin{aligned}
R_{b i} & =\text { resistencia de la probeta } i \\
R_{b m} & =\text { resistencia media de la serie. }
\end{aligned}
$$

Debemos desechar todas aquellas probetas cuya resistencia difiere de $\sigma_{b m}$ en $\pm 3 \delta$, ya que es tan pequeña su probabilidad de presentarse, caso de tener las probetas de la serie ensayada, la media $\sigma_{b m}$ y la desviación típica $\delta$ que debe existir alguna causa "no estadística" que haya provocado su aparición (ejecución de la probeta, curado, forma de rotura, transporte, etc.).

De todas formas, sea cual fuere el sistema empleado para caracterizar la resistencia de una serie de probetas es evidente que el sistema de «resistencia mínima admisible» no es adecuado estadísticamente, es imposible de cumplir desde el punto de vista teórico en un $100 \%$; y aún para probabilidades menores, exige a los hormigones medias mucho más altas que las necesarias.

Vamos, pues, a tratar de exponer el método a seguir para el tratamiento de los resultados de un conjunto de series de probetas, encaminado a obtener conclusiones apropiadas. 
En primer lugar, hemos de definir qué se va a considerar como resistencia de una muestra, si la media o la característica de la serie de probetas ejecutadas con ella, una vez definida esta resistencia supondremos que el hormigón fabricado a nuestro juicio es de características constantes, es decir, no podemos suponer, a priori, que va ha haber diferencias de una amasada a otra debido a que se mantienen constantes las granulometrías, tipos de áridos, forma de dosificación, cantidad de cemento, relación agua/cemento, etc. Las resistencias de distintas series de probetas pertenecen a una población que se distribuye siguiendo una ley normal de media $\sigma_{b m}$ y de desviación típica $\delta$. Si tomamos un conjunto de $n$ muestras (carecterizando cada una de ellas por el resultado del ensayo de series de $N$ probetas) sabemos que (Cf. Cramer: «Teoría de probabilidades y aplicaciones») llamando $\sigma_{b}$ a la resistencia obtenida en cada uno de los ensayos y $\delta$ a la desviación típica del conjunto de los ensayos:

$$
\begin{array}{ll}
Y=\frac{\sigma_{b}-\sigma_{b m}}{\delta}, & \text { se distribuye normalmente con media } 0 \text { y desviación típica } 1 . \\
Z=\frac{n \cdot S^{2}}{\delta^{2}}, & \quad \text { o sea, tiene una distribución } \chi^{2} \text { con } n-1 \text { grados de libertad. } \\
t=\sqrt{n-1} \cdot \frac{Y}{\sqrt{Z}}, & \text { tiene una distribución de Student de } n-1 \text { grados de libertad. }
\end{array}
$$

Teniendo en cuenta que una estimación de $\delta$ se obtiene mediante la fórmula

$$
\delta=\frac{\sqrt{n}}{\sqrt{n-1}} \cdot S,
$$

resulta que

$$
t=\sqrt{n-1} \cdot \frac{\frac{\sigma_{b}-\sigma_{b m}}{\delta}}{\sqrt{\frac{n \cdot S^{2}}{\delta^{2}}}}=\sqrt{\frac{n-1}{n}} \cdot \frac{\sigma_{b}-\sigma_{b m}}{S}=\frac{\sigma_{b}-\sigma_{b m}}{\delta_{\mathrm{est}}}
$$

tiene una distribución $t$ de Student con $n-1$ grados de libertad, siendo $n$ el número de ensayos empleados para calcular $S$ por la fórmula dada anteriormente.

Obtenida así la distribución de los valores de cada ensayo y definida la resistencia característica como aquello que tiene una posibilidad de $1: 5$ de no ser alcanzada, es fácil obtener la resistencia media necesaria del hormigón para que éste tenga al mismo tiempo la resistencia característica exigida en función del número de ensayos y del coeficiente de variación del hormigón ejecutado, definiéndose como coeficiente de variación el expresado en la igualdad:

$$
V=\frac{\delta_{\text {est }}}{\sigma_{b m}}
$$

de aquí se deduce que la relación entre resistencia media y característica viene indicada por la fórmula:

$$
\sigma_{b m}=\frac{\sigma_{b k}}{1-t \cdot V}
$$


siendo $t$ la «t de Student» para una probabilidad del $40 \%$ (1) y los grados de libertad indicados por el número de ensayos empleados en la estimación de $\sigma_{b m}$ y $V$ menos uno; según esto, $t$ toma los siguientes valores:

TA B L A I

\begin{tabular}{c|c|c|c|c|c|c|c|c|c|c|c|c|c|c}
\hline $\begin{array}{c}\text { Núm. de ensayos } \\
\text { menos 1 }\end{array}$ & $\mathbf{1}$ & $\mathbf{2}$ & $\mathbf{3}$ & $\mathbf{4}$ & $\mathbf{5}$ & $\mathbf{6}$ & $\mathbf{7}$ & $\mathbf{8}$ & $\mathbf{9}$ & $\mathbf{1 0}$ & $\mathbf{1 5}$ & $\mathbf{2 0}$ & $\mathbf{2 5}$ & $\infty$ \\
\hline$t$ & 1,376 & 1,061 & 0,978 & 0,941 & 0,920 & 0,906 & 0,896 & 0,889 & 0,883 & 0,879 & 0,866 & 0,860 & 0,856 & 0,842 \\
\hline
\end{tabular}

Si suponemos haber ejecutado un número suficientemente grande de ensayos el valor de

$$
\alpha=\frac{1}{1-t \cdot V}=\frac{1}{1-0,842 V},
$$

se puede resumir en la siguiente tabla:

T A B L A I I

\begin{tabular}{r|c|c|c|c|c|c|c|c|c}
\hline $\begin{array}{l}\text { Coeficiente } \\
\text { de variación (\%) }\end{array}$ & 5 & 7,5 & 10 & 12,5 & 15 & 17,5 & 20 & 22,5 & 25 \\
$\alpha=$ & 1,044 & 1,067 & 1,091 & 1,117 & 1,144 & 1,172 & 1,202 & 1,233 & 1,266 \\
\hline
\end{tabular}

A partir de estos datos se puede determinar la resistencia media que ha de alcanzar un hormigón para tener una resistencia característica determinada, teniendo en cuenta que la calidad de ejecución de un hormigón se relaciona con su coeficiente de variación, relación que podemos aproximar mediante la siguiente escala:

\section{COEFICIENTE DE VARIACION PARA DIFERENTES CALIDADES DE EJECUCION}

\begin{tabular}{c|c|c|c|c}
\hline Tipo de ejecución: & Excelente & Bueno & Suficiente & Malo \\
\hline Construcción normal & $\begin{array}{c}\text { por debajo de } \\
10 \%\end{array}$ & de 10 a $15 \%$ & de 15 a $20 \%$ & $\begin{array}{c}\text { por encima } \\
\text { de } 20 \%\end{array}$ \\
\hline
\end{tabular}

Estos valores son los indicados por el American Concrete Institute en el ACI Standard 214, «Recomended Practique for Evaluation of Compression Test Result of Field Concrete».

En su última edición (1965), se aplican estos valores al coeficiente de variación obtenido a partir de las medias de las series de probetas hechas con cada muestra ensayada.

Hasta esta edición, sin embargo, se aplicaban al coeficiente de variación del conjunto de todas las probetas, considerando como independientes las ejecutadas en una misma amasada.

La nueva concepción del ACI Standard 214-65 es lógica, dado que en sus anteriores ediciones se hacía notar que «las variaciones existentes entre las resistencias de las probetas de una misma serie es debida únicamente a la forma de fabricación, curado y métodos de rotura de las mismas.

(1) La tabulación de la " $t$ de Student" es tal que se han reflejado los valores $t_{p}$ para los que $P\left(|t|>t_{p}\right)=p \%$; entonces, si sólo un $20 \%$ de los valores han de estar por debajo de $t$, dada la simetría de la función, la proporción de valores tales que $|t|>t_{p}$ ha de ser el doble de este porcentaje, es decir, el $40 \%$ al que he hecho referencia. 
En este Standard hay un ejemplo en el cual se puede ver que para series de dos probetas el obtener el coeficiente de variación varía de 10,8 para las medias a 11,8 para todas las probetas.

En el ejemplo que se desarrolla más adelante se ha ejecutado el cálculo del coeficiente de variación en los dos casos.

El introducir las variaciones debidas al ensayo en las características del hormigón hace que se falsee la calidad del mismo, tanto más si el coeficiente de variación del hormigón fabricado es pequeño, ya que entonces la variación debida al ensayo influye mucho; también hace aumentar más el coeficiente de variación cuanto mayor es el número de probetas de cada serie.

De lo dicho anteriormente deducimos nuevamente la importancia de considerar la resistencia de una amasada caracterizada por un valor representativo del conjunto de probetas efectuado con la misma, olvidándose después de los resultados individuales para juzgar la calidad del hormigón, y empleándolos únicamente para deducir la calidad del ensayo efectuado.

El control a efectuar es el de la resistencia media y el coeficiente de variación de los resultados obtenidos para ver que se cumplen las condiciones previstas anteriormente; en una palabra, comprobar que el hormigón que se está fabricando tiene una resistencia característica igual o mayor que la especificada.

La primera dificultad que encontramos al implantar este tipo de control es el desconocimiento de la resistencia característica de un hormigón hasta que no existe un número de ensayos suficiente como para poder determinar con cierta exactitud el valor de la media y la desviación típica del hormigón ejecutado.

Suponiendo un coeficiente de variación del $15 \%$ obtenido de un número suficiente de ensayos, para lograr que el error en la estimación de la media no sea mayor del $5 \%$ con una probabilidad de 0,9 , el número de ensayos necesarios será (según las fórmulas antes indicadas):

$$
n=\left(\frac{\lambda_{p} \cdot V}{E}\right)^{2}=\left(\frac{1,645 \times 15}{5}\right)=24,4 \sim 25 \text { series; }
$$

entonces el valor de $\delta$ se estima en:

$$
\sqrt{\frac{n}{n-1}} \cdot S=\sqrt{\frac{25}{24}} \cdot S=1,04 S
$$

con un intervalo de confianza:

$$
\frac{\sqrt{n}}{\chi_{p}} \quad \text { a } \cdot \frac{\sqrt{n}}{\chi_{p}^{\prime}} \cdot S
$$

es decir, de $0,84 S$ a $1,34 S$ con una probabilidad del $90 \%$, aproximación que podemos considerar como suficiente.

Es, por tanto, necesario esperar bastante tiempo para tener suficiente confianza en la obtención de la resistencia característica; sin embargo, con un número menor de series de probetas (aproximadamente 10) podemos comenzar un estudio estadístico que nos vaya informando de las tendencias que siguen las resistencias del hormigón confeccionado. 
El proceso a seguir es el siguiente:

a) Llevar un control de las resistencias representativas de cada serie; en este control existirán los siguientes límites:

1. Resistencia media estimada como necesaria $\sigma_{b m}$.

2. Resistencia característica exigida $\sigma_{b k}$.

3. Los valores $\sigma_{b m}(1-2 V)$ y $\sigma_{b m}(1-3 V)$, en donde $V$ es el coeficiente de variación estimado para el hormigón ejecutado, el valor de $V$ debe corregirse en el momento de tener datos suficientes corrigiéndose automáticamente $\sigma_{b m}$ y los límites indicados en 3 .

Suponiendo una distribución normal en los resultados de los ensayos realizados, resulta que las probabilidades de obtener en los ensayos 1, 2, 3, etc. resultados consecutivos menores que

$$
\sigma_{b k} \quad ; \quad \sigma_{b m}(1-2 V) \quad \text { y } \quad \sigma_{b m}(1-3 V)
$$

son las siguientes:

T A B L A I I I

\begin{tabular}{c|l|c|c|c}
\hline $\begin{array}{c}\text { Número de probetas con- } \\
\text { secutivas de valor menor } \\
\text { que el indicado }\end{array}$ & $\sigma_{b k}$ & $\sigma_{b m}(1-V)$ & $\sigma_{b m}(\mathbf{1}-\mathbf{2} \mathbf{V})$ & $\sigma_{b m}(1-\mathbf{3} \mathbf{V})$ \\
\hline 1 & 0,21 & 0,16 & 0,023 & 0,0014 \\
2 & 0,044 & 0,0256 & 0,0005 & - \\
3 & 0,00924 & 0,004 & - & - \\
4 & 0,002 & 0,00064 & - & - \\
\hline
\end{tabular}

Estas probabilidades están obtenidas suponiendo la población dividida en dos grupos, uno de valor igual o mayor que el indicado y otro de valor menor que el indicado; el problema se reduce entonces al de extracciones con reposición, y la probabilidad de que aparezcan en $1,2,3,4, \ldots, n$ extracciones todos los elementos del mismo grupo es:

$$
P_{n}=\gamma^{n},
$$

siendo $\gamma$ el porcentaje de elementos del grupo elegido dentro de la población estudiada, y que se obtiene como $1 / 2$ del valor de la probabilidad de que en una distribución normal $P\left(|x-m|>\lambda_{p} \cdot \delta\right)=\gamma \%$.

Podemos, ahora, partiendo de las probabilidades antes obtenidas, establecer el siguiente criterio:

T A B L A I V

\begin{tabular}{c|c|c|c|c}
\hline \multicolumn{1}{c|}{ Valor límite } & $\sigma_{b k}$ & $\sigma_{b m}(1-V)$ & $\sigma_{b m}(1-2 V)$ & $\sigma_{b m}(1-3 V)$ \\
\hline $\begin{array}{l}\text { Número de valores por } \\
\text { debajo del límite que } \\
\text { hacen dudar }\end{array}$ & 3 & 2 & 1 & - \\
$\begin{array}{l}\text { Número de ensayos por } \\
\text { debajo del límite que }\end{array}$ & 4 & 3 & 2 & 1 \\
$\begin{array}{l}\text { indican incumplimiento } \\
\text { de las especificaciones }\end{array}$ & 4 & & \\
\hline
\end{tabular}


Esto da una indicación de la marcha del hormigón sobre todo a los primeros días, ayudando a juzgar si encaja o no en las previsiones hechas.

b) Otro elemento útil en la consecución de un control ágil es el gráfico de medias de las medias.

Si la población a que pertenecen las resistencias obtenidas hemos supuesto que es normal $\left(\sigma_{b m}, \delta\right)$, la media de $n$ resultados sigue a su vez una distribución normal de media $\sigma_{b m}$ y desviación típica $\delta / \sqrt{n}$.

Por otro lado, ya hemos dicho (Cf. Pedro Mendizábal: «El Control Estadístico de Calidad», págs. 37 y ss.) que normalmente se considera como valores límites, es decir, valores que tomados por la variable indican que es necesario buscar una causa «asignable» que justifique la aparición de este valor si realmente la población sigue una distribución normal $\left(\sigma_{b m}, \delta\right)$, a los valores $\left(\sigma_{b m}-3 \delta\right)$, y como valores de alerta, es decir, aquellos que nos hacen dudar, al aparecer, de la realidad de las hipótesis hechas respecto a los parámetros de la distribución, a los valores $\left(\sigma_{b m}-2 \delta\right)$. Ya que sólo tenemos interés en los valores menores que los establecidos.

Suponiendo, pues, un coeficiente de variación $V$, los valores límite y de alerta para la media de $n$ valores serán:

$$
A=\sigma_{b m}\left(1-\frac{2 V}{\sqrt{n}}\right), \quad B=\sigma_{b m}\left(1-\frac{3 V}{\sqrt{n}}\right) ;
$$

relacionando estos valores con $\sigma_{b k}$ nos resulta la siguiente relación de valores para $V=15 \%$, y siendo:

$$
\alpha_{1}=\frac{A}{\sigma_{b k}} \quad \mathrm{y} \quad \alpha_{2}=\frac{B}{\sigma_{b k}}
$$

T A B L A V

\begin{tabular}{c|c|c}
\hline $\begin{array}{c}\text { Número de ensayos de los que } \\
\text { se obtiene la media }\end{array}$ & $\alpha_{1}$ & $\alpha_{2}$ \\
\hline 1 & 0,8 & 0,625 \\
2 & 0,9 & 0,77 \\
3 & 0,94 & 0,84 \\
4 & 0,97 & 0,89 \\
5 & 0,99 & 0,91 \\
6 & 1,00 & 0,93 \\
Probabilidad de obtener un & $2,25 \%$ & $0,14 \%$ \\
valor menor que $\alpha \sigma_{b k}$ & & \\
\hline
\end{tabular}

Si se lleva este control mediante gráficos, se dibujan en ellos las líneas $\alpha_{1} \cdot \sigma_{b k} \mathrm{y}$ $\alpha_{2} \cdot \sigma_{b k}$. Si aparecen valores por debajo de la primera línea, hay riesgo de que no cumpla los especificaciones. Caso de obtener valores por debajo de la segunda línea, se puede suponer que existe garantía de que el hormigón no cumple las condiciones previstas. Si aparece un solo resultado fuera de estas líneas, se debe buscar la causa del descenso de resistencia; pues si se encuentra y no es achacable al hormigón sino a la manipulación de las probetas o al ensayo, hará que se deseche este resultado sin influir en la caracterización del hormigón fabricado. Caso de estar la causa en el hormigón, habrá que tomar las medidas oportunas para conseguir que éste cumpla las especificaciones propuestas. 
c) Otro control interesante, sobre todo cuando no se tiene conocimiento de la forma de fabricación de cada $\mathrm{m}^{3}$ de hormigón o no se tiene acceso a las plantas dosificadoras de áridos... (como es en el caso de emplear en obra hormigones premezclados) es de las resistencias características de 10 ensayos consecutivos. Este control se efectúa por el método aproximado de la media de la mitad más baja de las resistencias y sirve para controlar la uniformidad del hormigón fabricado a lo largo del tiempo, ya que pueden existir variaciones en la dosificación, en la confección, etc., que hagan variar la resistencia durante cierto período; esta curva detecta las variaciones en la resistencia, debidas a causas no estadísticas. No se ha de obligar a esta media a ser superior a la resistencia característica exigida; pero si aparecen de forma continuada valores por debajo de $\sigma_{b k}$, se ha de hacer un estudio especial con el hormigón fabricado en el período a que estas medidas se refieren para ver si realmente en este período no se alcanzó la resistencia característica exigida, a pesar de que puede ocurrir que, para el conjunto de todo el hormigón del tipo estudiado, fuera la resistencia característica superior a la exigida.

Este mismo defecto se acusa en la curva de media de las medias. En ella se pueden ver las zonas en que el hormigón ha bajado de resistencia respecto al resto y estudiar estos períodos de forma diferentes; sin embargo, de esta forma es a veces necesario estudiar períodos que en la curva de resistencias características de 10 probetas consecutivas dan resistencia por encima de la exigida.

Hay que tener en cuenta, no obstante, que pequeñas disminuciones de la resistencia característica en períodos concretos no debe hacer pensar que el hormigón de este período no cumple las especificaciones si lo cumple todo el conjunto de ensayos, ya que estas pequeñas variaciones pueden deberse a motivos puramente estadísticos.

La interpretación de este gráfico ha de hacerse, por tanto, con gran cuidado; pero, sabiéndole dar la importancia necesaria en cada caso, puede ser de gran interés.

Como un dato de interpretación podemos hacer el siguiente cálculo. La media de 10 ensayos consecutivos se distribuirá normalmente: una media $\sigma_{b m}$ y desviación típica $\delta / \sqrt{10}$; resultará, entonces, que hay una probabilidad de 0,20 de que se obtenga para esta media un valor menor que:

$$
\sigma_{b m}=\frac{0,8 \delta}{3,16},
$$

y la resistencia característica que obtendremos en un $20 \%$ de los casos será menor que

$$
\sigma_{b m}-\frac{0,3}{3,16} \times \frac{0,88}{1}=\sigma_{b m}-1,13 \delta<\sigma_{b k} ;
$$

es, pues, bastante probable encontrar valores menores que $\sigma_{b k}$ en la resistencia característica de 10 ensayos consecutivos; se ha de estudiar, por lo tanto, la tendencia de la curva sin preocuparse de la aparición de valores menores que la característica exigida.

d) Por último es de gran interés poder estimar la calidad de los ensayos realizados, o sea, la aproximación con que estamos midiendo la resistencia de cada muestra ensayada, calidad que podemos apreciar mediante la desviación típica de las series de probetas, es decir, la dispersión en la medida del valor único que es la resistencia de una serie de probetas ejecutadas debidamente.

Para series de un número de probetas normal en la construcción, es decir, que no supera a 10 , pero que está normalmente entre 3 y 6 , la representación de la varia. bilidad se hace mediante el "recorrido» o diferencia entre el valor mayor y menor de los obtenidos en la serie de probetas. 
Suponiendo una distribución normal, se puede esperar una relación entre la desviación típica $(\delta)$ y el recorrido $(R)$ tal que:

$$
\delta=\frac{1}{d_{n}} \cdot R .
$$

TA B L A V I

FACTORES QUE RELACIONAN LA DESVIACION TIPICA DEL ENSAYO Y EL RECORRIDO

\begin{tabular}{c|c|c}
\hline $\begin{array}{c}\text { Número de probetas } \\
\text { de la serie }\end{array}$ & $\boldsymbol{d}_{\boldsymbol{n}}$ & $\boldsymbol{1}$ \\
\hline 2 & 1,128 & $\boldsymbol{d}_{\boldsymbol{n}}$ \\
3 & 1,693 & 0,8865 \\
4 & 2,059 & 0,5907 \\
5 & 2,326 & 0,4857 \\
6 & 2,534 & 0,4299 \\
7 & 2,704 & 0,3946 \\
8 & 2,847 & 0,3698 \\
9 & 2,970 & 0,3512 \\
10 & 3,078 & 0,3367 \\
\end{tabular}

Supondremos ahora que el ensayo tiene una dispersión propia y que ésta puede estimarse con la media de 10 recorridos consecutivos; suponiendo, a su vez, que esta dispersión es tal que el coeficiente de variación máxima es de un $5 \%$ sobre la media general del hormigón fabricado, podemos estimar un valor máximo del recorrido, compatible con las hipótesis efectuadas respecto al hormigón y a la calidad del ensayo.

En este caso:

$$
R_{\max }=d_{n} \cdot \sigma_{b m} \cdot V=0,05 \sigma_{b m} \cdot d_{n} ;
$$

fórmula que indicará el valor máximo de la media de 10 recorridos consecutivos.

La forma de trabajo puede normalizarse en los impresos del ejemplo adjunto. Mediante ellos podemos llevar el control diario y obtener los coeficientes de variación, desviación típica, resistencia característica, etc. En dicho ejemplo, que indicaremos a continuación, aplicaremos el método numérico y el método gráfico con papel probabilístico comparando ambos resultados.

Este ejemplo irá mostrando el proceso práctico a seguir y nos indicará, además, cómo varían las resistencias así controladas en la realidad.

Ejemplo.-Hormigón para el tablero del paso superior núm. 2 en el Nudo de Villalba:

Hormigón: de $350 \mathrm{~kg}$ de cemento P-450;

Arido: arena de río y granito machacado.

Se efectuaba un control regular de la granulometría de los áridos que se dosificaban en dos tamaños, arena de $0-5$ y granito machacado de 5 a 25 .

Los resultados obtenidos se resumen en la siguiente tabla: 
T A B L A E - I

\begin{tabular}{|c|c|c|c|c|c|}
\hline $\begin{array}{l}\text { Número } \\
\text { de la probeta }\end{array}$ & $\begin{array}{l}\text { Fecha } \\
\text { de ejecución }\end{array}$ & $\begin{array}{c}7 \text { dias (media } \\
\text { de } \\
2 \text { probetas) }\end{array}$ & Recorrido & $\begin{array}{c}\text { Media de } 6 \\
\text { recorridos } \\
\text { consecutivos }\end{array}$ & $\begin{array}{c}\text { Media de } 10 \\
\text { recorridos } \\
\text { consecutivos }\end{array}$ \\
\hline \multirow{5}{*}{$\begin{array}{l}\text { 21-PNV-45 } \\
\text { 21-PNV-45-O. P. } \\
\text { 22-PNV45 } \\
\text { 23-PNV-45 } \\
\text { 23-PNV-45-O. P. }\end{array}$} & $27-10-66$ & 373 & 0 & - & - \\
\hline & $27-10-66$ & $\begin{array}{l}385 \\
263\end{array}$ & 0 & $\overline{-}$ & - \\
\hline & $\begin{array}{l}21-10-60 \\
28-10-66\end{array}$ & $\begin{array}{l}203 \\
337\end{array}$ & $\begin{array}{l}40 \\
51\end{array}$ & - & - \\
\hline & $28-10-66$ & 315 & 17 & - & - \\
\hline & $29-10-66$ & 349 & 9 & 337 & - \\
\hline O. P. & $29-10-66$ & 386 & 11 & 339 & - \\
\hline 25 & $31-10-66$ & 376 & 40 & 338 & - \\
\hline 26 & $1-11-66$ & 356 & 79 & 353 & $\overline{0}$ \\
\hline 27 & $2-11-66$ & 299 & 55 & 347 & 30 \\
\hline 28 & $3-11-66$ & 336 & 39 & 350 & 34 \\
\hline O. P. & $3-11-66$ & 346 & 11 & $\begin{array}{l}350 \\
353\end{array}$ & $\begin{array}{l}35 \\
25\end{array}$ \\
\hline 29 & $5-11-66$ & 404 & 39 & $\begin{array}{l}353 \\
324\end{array}$ & $\begin{array}{l}35 \\
32\end{array}$ \\
\hline 30 & $\begin{array}{l}9-11-66 \\
9-11-66\end{array}$ & $\begin{array}{l}309 \\
348\end{array}$ & $\begin{array}{l}11 \\
17\end{array}$ & $\begin{array}{l}334 \\
334\end{array}$ & 32 \\
\hline $\begin{array}{l}30 \\
31\end{array}$ & $\begin{array}{r}9-11-66 \\
10-11-66\end{array}$ & 356 & 45 & 335 & $\begin{array}{l}52 \\
35\end{array}$ \\
\hline O. P. & $10-11-66$ & 306 & 46 & 345 & 39 \\
\hline 32 & $11-11-66$ & 354 & 5 & 346 & 35 \\
\hline O.P. & $11-11-66$ & 365 & 17 & 340 & 29 \\
\hline 33 & $12-11-66$ & 359 & 6 & 348 & 24 \\
\hline O. P. & $12-11-66$ & 357 & 0 & 350 & 20 \\
\hline 34 & $14-11-66$ & 410 & 62 & 358 & 25 \\
\hline O. P. & $14-11-66$ & 374 & 12 & 370 & 23 \\
\hline 35 & $15-11-66$ & 317 & 11 & 364 & 22 \\
\hline 36 & $16-11-66$ & 386 & 3 & 367 & 21 \\
\hline 37 & $17-11-66$ & $\begin{array}{l}359 \\
230\end{array}$ & 17 & 367 & 18 \\
\hline $\begin{array}{l}38 \\
39\end{array}$ & $\begin{array}{l}18-11-66 \\
21-11-66\end{array}$ & $\begin{array}{l}339 \\
338\end{array}$ & $\begin{array}{l}68 \\
17\end{array}$ & $\begin{array}{l}364 \\
352\end{array}$ & $\begin{array}{l}20 \\
21\end{array}$ \\
\hline 40 & $22-11-66$ & 334 & 45 & 346 & 24 \\
\hline 41 & $23-11-66$ & 362 & 22 & 353 & 26 \\
\hline 42 & $24-11-66$ & 328 & 12 & 343 & 27 \\
\hline
\end{tabular}

TABLA E-I I

\begin{tabular}{|c|c|c|c|c|c|}
\hline $\begin{array}{l}\text { Número } \\
\text { de la probeta }\end{array}$ & $\begin{array}{c}28 \text { días } \\
\text { (media de } 6 \\
\text { probetas) }\end{array}$ & Recorrido & $\begin{array}{c}\text { Media de } 6 \\
\text { días } \\
\text { consecutivos }\end{array}$ & $\begin{array}{c}\text { Media de } 10 \\
\text { días } \\
\text { consecutivos }\end{array}$ & $\begin{array}{c}\text { Media de } 10 \\
\text { rangos }\end{array}$ \\
\hline 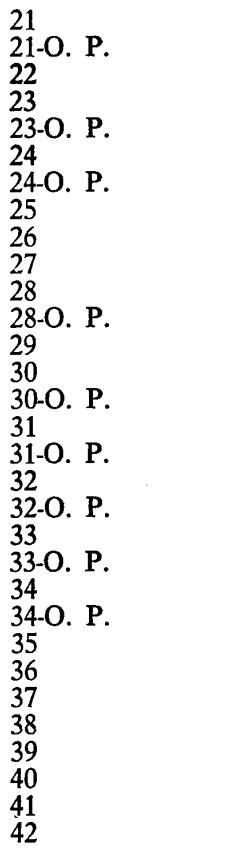 & $\begin{array}{l}481 \\
458 \\
406 \\
446 \\
454 \\
475 \\
521 \\
510 \\
500 \\
458 \\
500 \\
530 \\
528 \\
495 \\
437 \\
460 \\
390 \\
445 \\
462 \\
490 \\
436 \\
490 \\
438 \\
468 \\
460 \\
512 \\
501 \\
464 \\
496 \\
492 \\
464\end{array}$ & $\begin{array}{r}79 \\
34 \\
34 \\
46 \\
79 \\
79 \\
68 \\
113 \\
107 \\
62 \\
49 \\
16 \\
33 \\
50 \\
51 \\
78 \\
45 \\
73 \\
40 \\
130 \\
141 \\
113 \\
146 \\
51 \\
140 \\
57 \\
68 \\
90 \\
67 \\
39 \\
67\end{array}$ & $\begin{array}{l}- \\
- \\
- \\
\overline{-} \\
453 \\
460 \\
469 \\
484 \\
486 \\
494 \\
503 \\
504 \\
502 \\
491 \\
492 \\
473 \\
458 \\
447 \\
447 \\
452 \\
460 \\
464 \\
464 \\
467 \\
478 \\
474 \\
483 \\
487 \\
488 \\
486\end{array}$ & $\begin{array}{l}- \\
- \\
- \\
- \\
- \\
- \\
- \\
444 \\
444 \\
448 \\
463 \\
476 \\
473 \\
470 \\
448 \\
438 \\
438 \\
439 \\
434 \\
434 \\
429 \\
429 \\
434 \\
434 \\
448 \\
452 \\
453 \\
453 \\
459\end{array}$ & $\begin{array}{l}\text { - } \\
\text { - } \\
- \\
- \\
- \\
- \\
71 \\
67 \\
66 \\
66 \\
67 \\
64 \\
64 \\
61 \\
57 \\
51 \\
57 \\
67 \\
76 \\
88 \\
88 \\
97 \\
95 \\
97 \\
99 \\
101 \\
92 \\
85\end{array}$ \\
\hline
\end{tabular}


Los valores obtenidos en las tablas I y II son las medias de cada ensayo de 2 y 6 probetas, respectivamente. Todo el tratamiento de estos datos los efectuamos en las figuras 1, 2, 3 y 4; al mismo tiempo hemos obtenido numéricamente los valores de $\sigma_{b m}, \delta, V$, y el de $\sigma_{b k}$ para poder compararlos con los obtenidos en el método simplificado seguido en las figuras 3 y 4 , y en el método gráfico seguido en los figuras 5 y 6. Estos valores son:

Resultados a 7 días

$$
\sigma_{b m}=\frac{10.826}{31}=349
$$

Recorrido medio $R=26,2$.

Desviación típica del ensayo $=0,8865 \times 26,2=23,23$.

Coeficiente de variación del ensayo $=\delta / \sigma_{b m}=6,7 \%$.

Resultados a 28 días

$$
\sigma_{b m}=474 \mathrm{kp} / \mathrm{cm}^{2} .
$$

Recorrido medio $=72 \mathrm{kp} / \mathrm{m}^{2}$.

Coeficiente de variación del hormigón $=6,9 \%$.

Coeficiente de variación del ensayo $=5,95 \%$.

Resistencia característica del hormigón $=474(1-0,84 \times 0,069)=446 \mathrm{kp} / \mathrm{cm}^{2}$.

Para comparar este estudio con los resultados obtenidos al considerar todas las probetas (incluso los de una misma clase) como independientes para determinar la calidad del hormigón se han efectuado los cálculos correlativos en las figuras 4 y 6 , así como cálculos equivalentes cuyos resultados damos a continuación:

$\sigma_{b m}=474 \mathrm{kp} / \mathrm{cm}^{2}$.

Coeficiente de variación del hormigón $=0,3 \%$.

$\sigma_{b k}=474(1-0,84 \times 0,093)=437 \mathrm{kp} / \mathrm{cm}^{2}$.

Estos resultados, al compararlos con los obtenidos por los otros métodos, nos indican la aproximación de los mismos.

De los gráficos de control se deduce fácilmente que el hormigón ejecutado ha estado siempre dentro de las especificaciones, con excepción de la calidad del ensayo que no ha sido suficientemente buena. Para el control del hormigón a 7 días se exigió una resistencia media de valor $\sigma_{b m} \times 0,75$, por suponerse que ésta iba a ser la relación entre la resistencia a 7 y a 28 días en la época en que se efectuó el hormigonado.

\section{conclusiones}

1. El control de hormigones para su resistencia a compresión debe hacerse a partir de muestras representadas por el valor medio de las probetas ejecutadas con ellas.

2. Para esta caracterización bastan series de 3 probetas.

3. En estas series deben rechazarse como defectuosas todas aquellas probetas que rompan con valores superiores a $R_{b m} \pm 3 \delta$, siendo $R_{b m}$ la resistencia media de la serie.

4. El control de calidad se efectúa mediante la media y el coeficiente de variación obtenido del conjunto de valores medios representativos de cada serie.

5. La obtención de la media y la resistencia característica se puede efectuar de forma rutinaria en los impresos que se muestran en el ejemplo. 


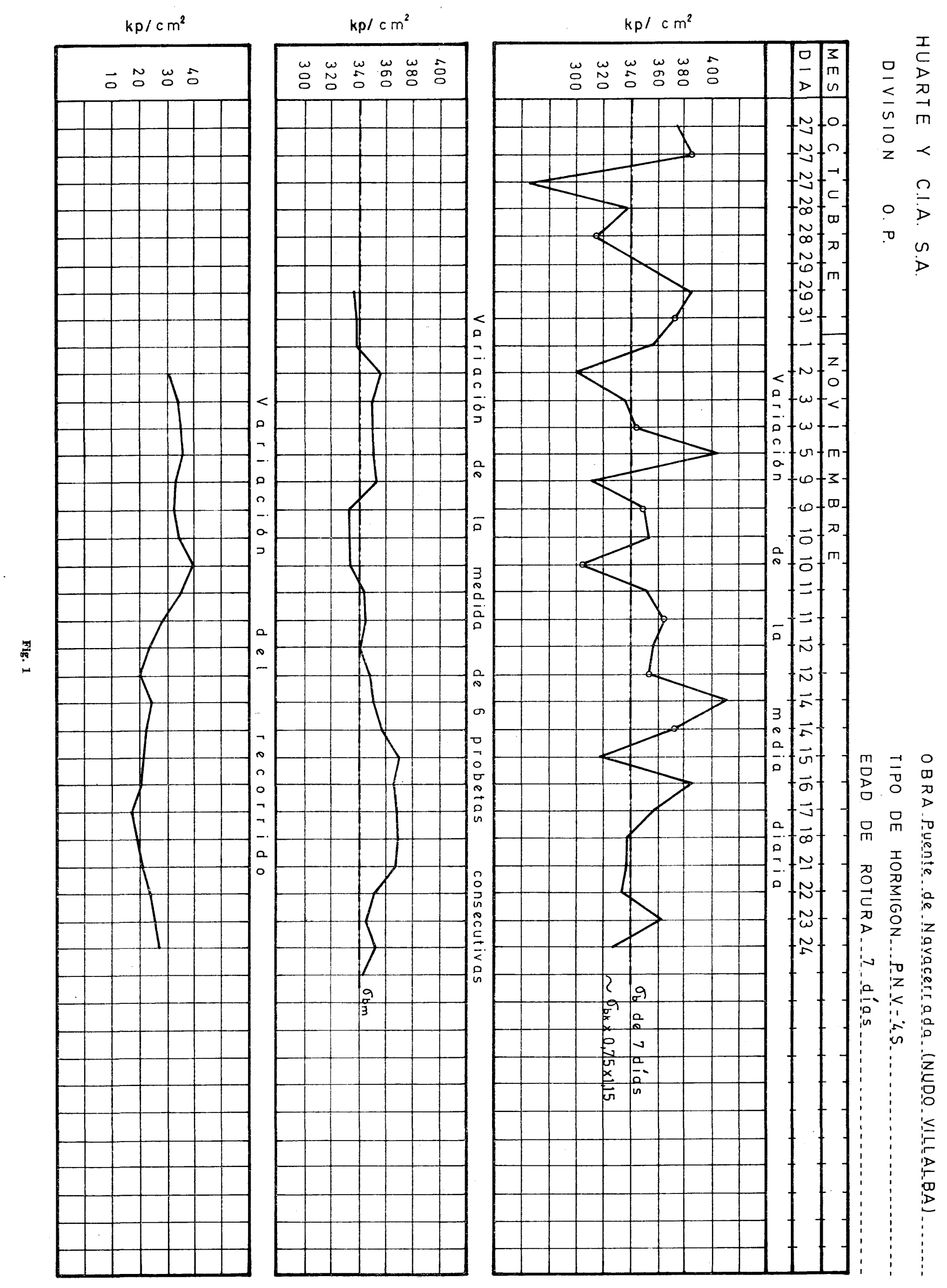




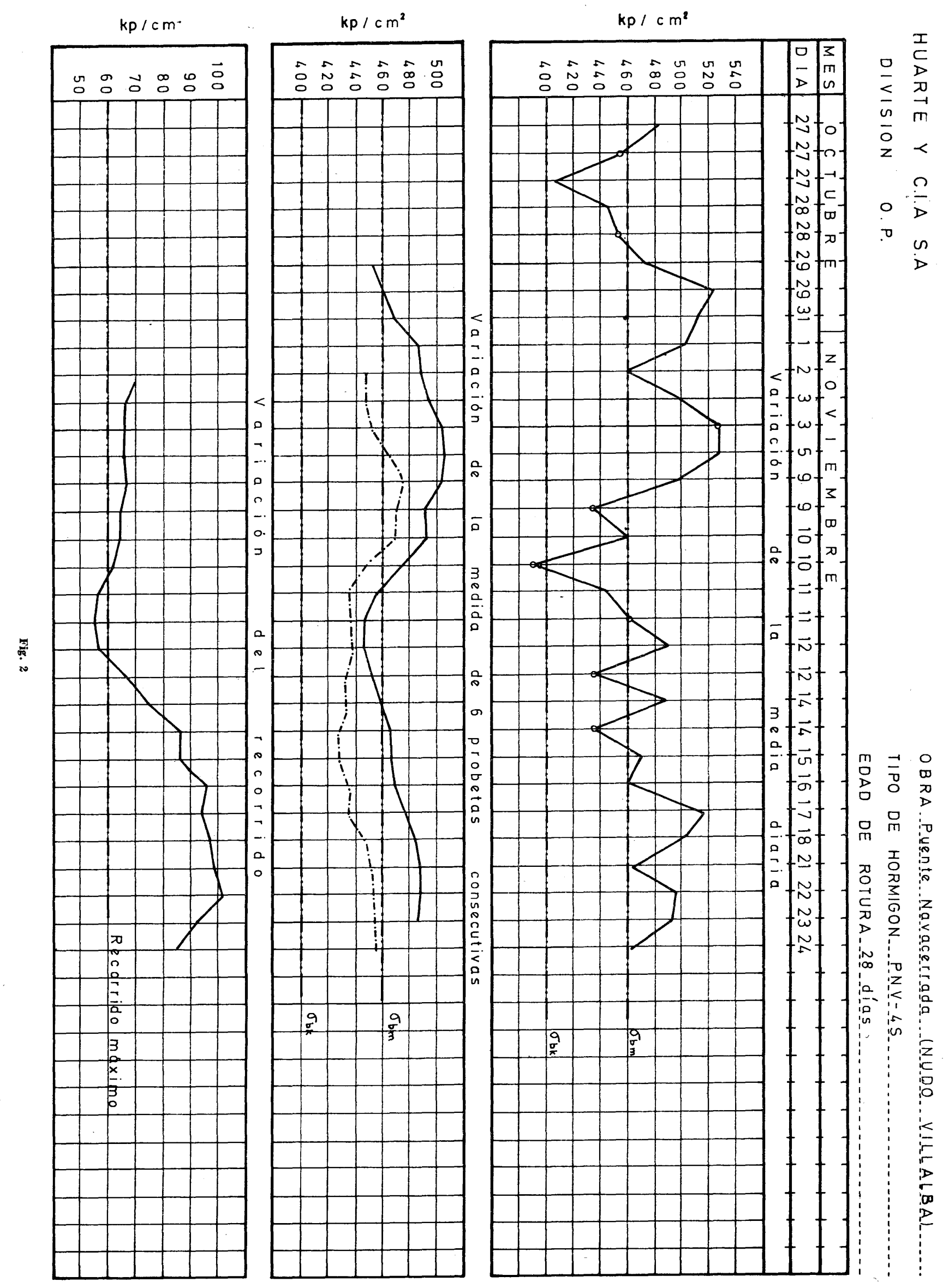




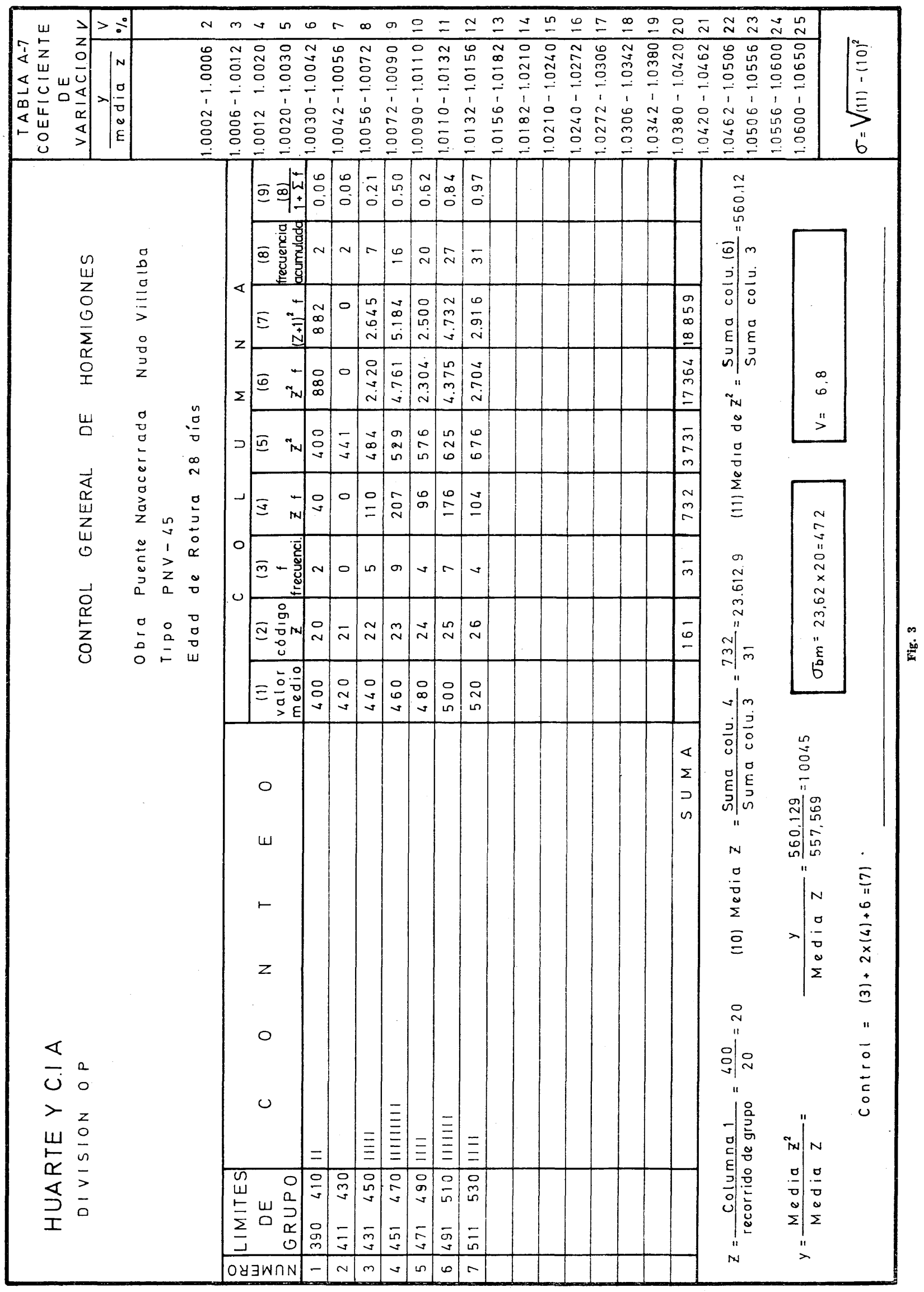




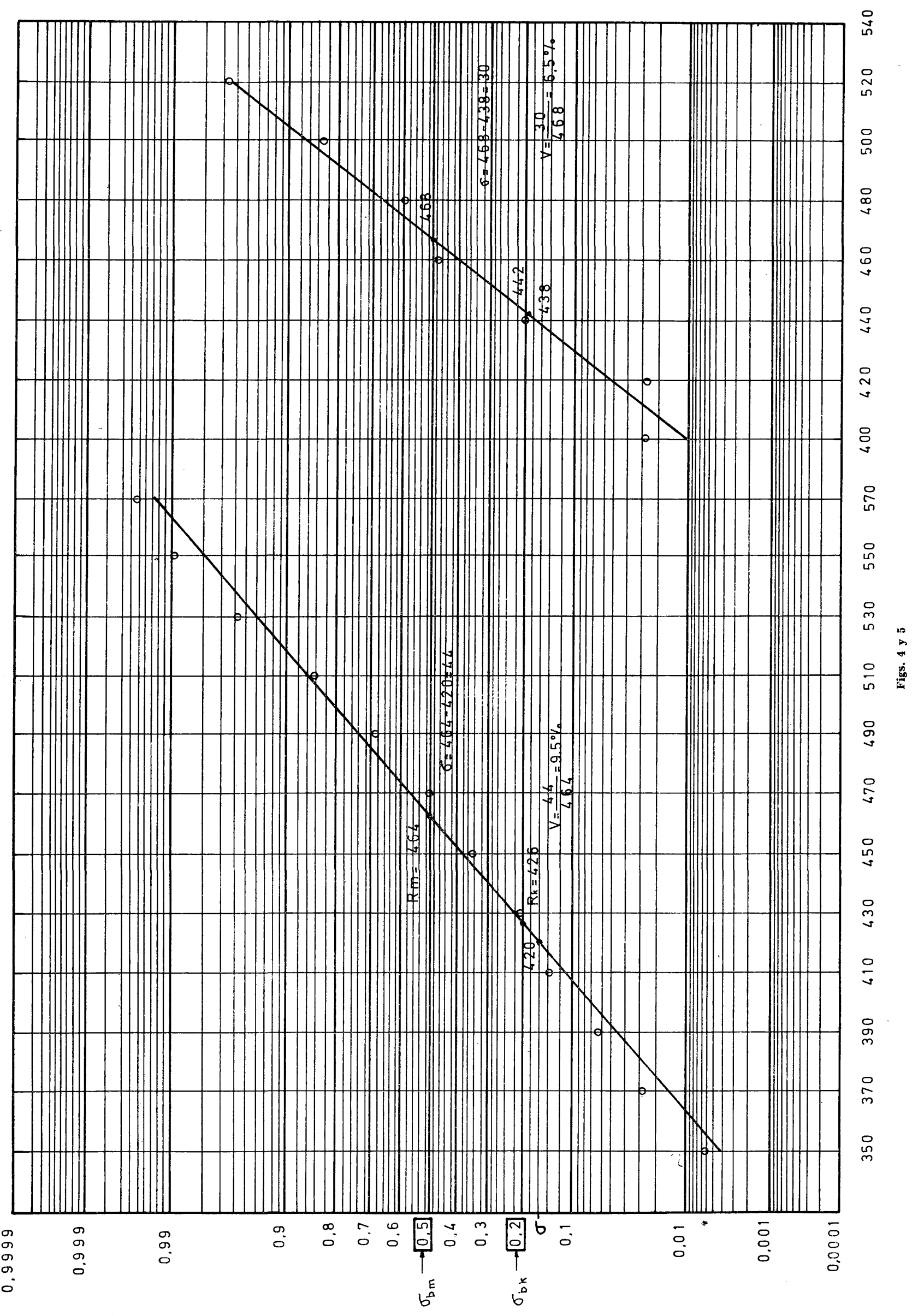




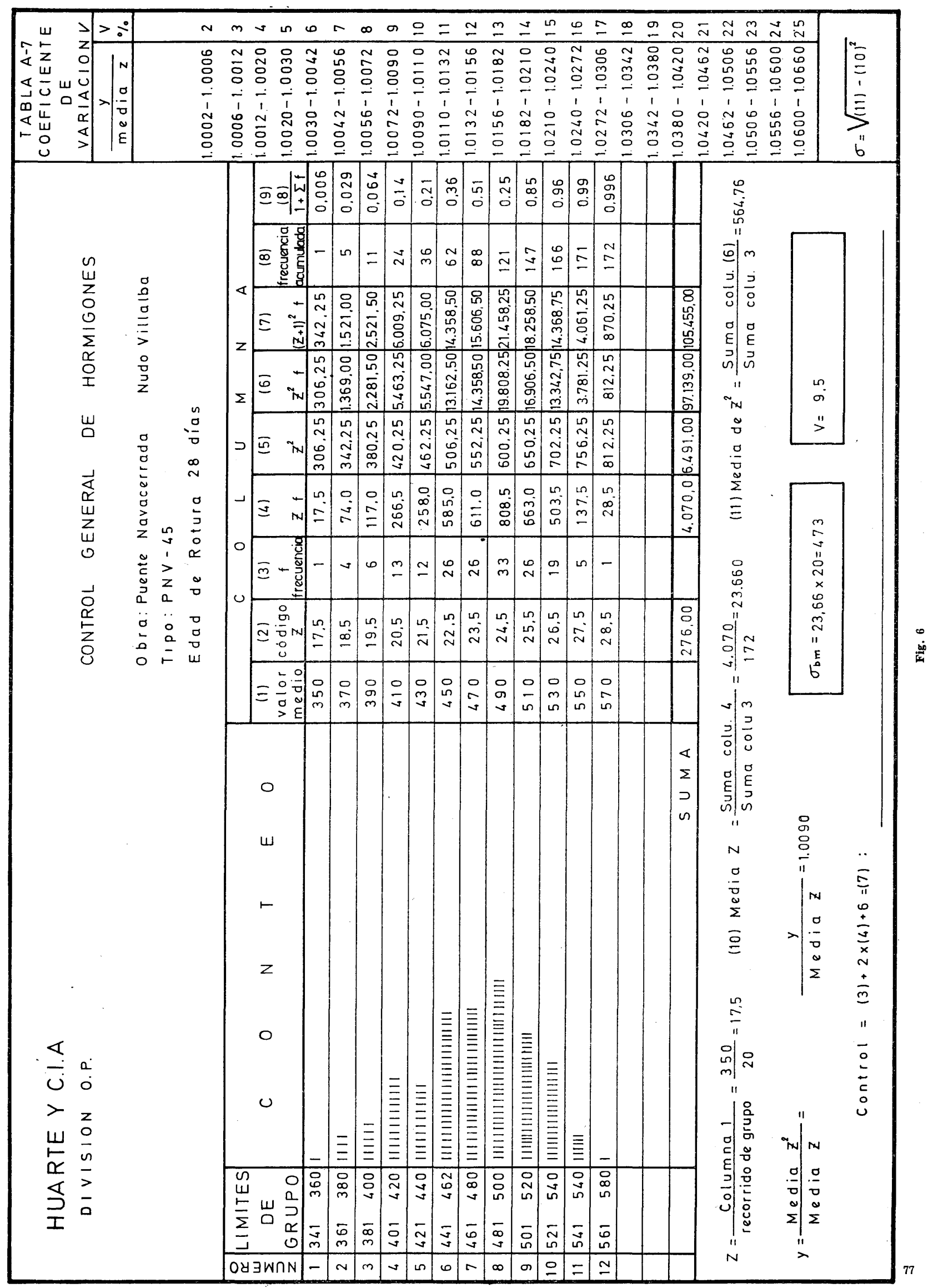




\section{and}

En el intenalo entre la escritura de este articulo y su publicación ha aparecido la Insthuc ción para el proyećto y la ejecúción de las obras de hormigón en masa o armado del $M O P$. (1968) En ella se define el valor de ra resistencia característica de fóma distinta que en. el $H: 61$.

En función de la resistencia media y del cóficente de variacono ta résistencia característica se define en este caso como:

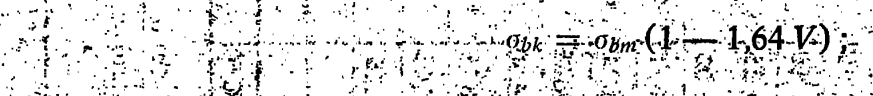

para esta nueva defnión varín tôs valores de las diversas táblas por ello qucremos indicar aquilsu varacion para poder estudian un sistema de control en el caso de emplearse esta nueva definicion de resistencia caractéstica.

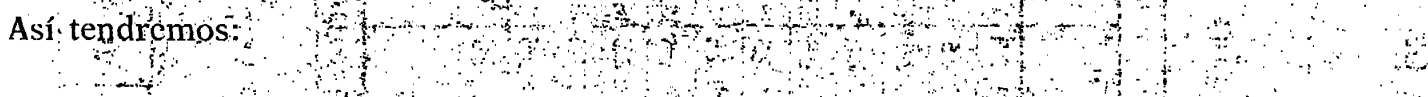

\begin{tabular}{|c|c|c|c|c|c|c|c|c|c|c|c|c|c|}
\hline $\begin{array}{l}\text { No de ensayos } \\
\text { menos } 1\end{array}$ & 1 & 3 & $4^{-}$ & 5 & 66 & 7 & 8 & 9 & 10 & 15 & 20 & 25 & $\infty$ \\
\hline$t$ & $6,31 / 292$ & 2,35 & 22,31 & 2,02 & 1,24 & 189 & 1,86 & 1,83 & 1,8 & 1,75 & 1,72 & 1,71 & 164 \\
\hline
\end{tabular}

TABLA I I

\begin{tabular}{|c|c|c|c|c|c|c|c|c|}
\hline $\begin{array}{l}\text { Coeficiente } \\
\text { de variación }\end{array}$ & 75 & ii) & $\mathrm{i} 2,5$ & 15 & 17,5 & 20 & 22,5 & 25 \\
\hline$\alpha$ & $1 ; 14$ & 1,20 & 1,26 & $1 ; 33$ & 1,40 & 1,49 & 1,58 & 1,70 \\
\hline
\end{tabular}

.

TABLA III

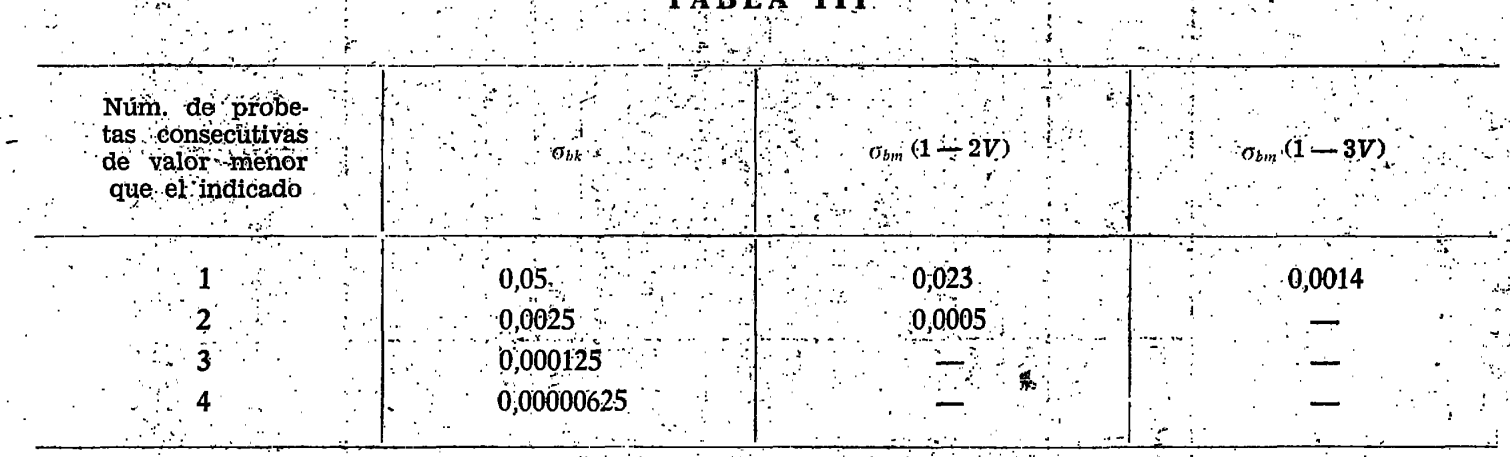


T A B L A I V

\begin{tabular}{l|c|c|c}
\hline Valor límite & $\sigma_{b k}$ & $\sigma_{b m}(1-2 V)$ & $\sigma_{b m}(1-3 V)$ \\
\hline $\begin{array}{l}\text { Núm. de valo- } \\
\text { res por debajo } \\
\text { del límite que } \\
\text { hacen dudar }\end{array}$ & 1 & 1 & - \\
$\begin{array}{l}\text { Núm. de ensa- } \\
\text { yos por debajo } \\
\text { del límite que } \\
\text { indica incum- } \\
\text { plimiento de } \\
\text { las especifica- } \\
\text { ciones }\end{array}$ & 3 & & \\
\hline
\end{tabular}

Por último, en esta instrucción el artículo 23.4 halla la forma de ejecutar el control. Sin embargo, se cae también en el defecto de efectuar en control de valor mínimo, por ello ha de insistir de nuevo en las conclusiones anteriores, indicando que las probetas ejecutadas de una sola amasada representa en valor único y que la resistencia característica ha de calcularse sobre el conjunto total de amasadas controladas y que se han ejecutado de la misma forma y con la misma dosificación.

\section{mésumé: 0 summmary 0 zusammmenfassinng}

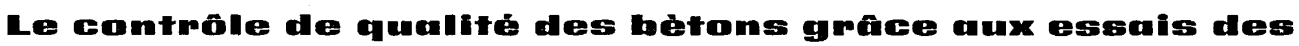
Ǵprouvettes am coumpression simpiole

José M. López Saiz, ingénieur des Ponts et Chaussées, A. M. ASCE

Cet article expose quelques idées au sujet du contrôle statistique de la qualité des bétons employés dans les travaux. Les tendances actuelles de ce contrôle sont commentées et éclaircies par un exemple pris sur un ouvrage réel, le pont de Navacerrada au noeud routier de Villalba exécuté par Huarte et Cie, $S$. A. et dont le projet est dû à l'ingénieur Carlos Fernández Casado qui en a amplement informé dans un article inséré dans cette revue.

\section{Quality Bontmol of Bomcrotes be Testimg of Sperimmems under Simmple Bommpession}

José M. López Saiz, Civil Engineer, A. M. ASCE

This paper presents some ideas on the statistical quality control of concrete used at working sites. Comments are offered on present tendencies in this type of control; an example being taken from an actual case, namely the Navacerrada Bridge at the Villalba Interchange, which was constructed by Huarte \& Cia., S. A., to the design of Mr. Carlos Fernández Casado. This project was commented in this magazine.

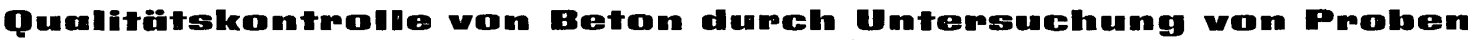 unter einforkheme Druck}

José M. López Saiz, Wege-, Kanal- und Hafenbauingenieur, A. M. ASCE

Der vorliegende Artikel erläutert einige Gedanken zur statistischen Kontrolle der Qualität des für Bauten verwendeten Betons. Es werden die gegenwärtigen Kontrollverfahren behandelt und anhand eines aus der Praxis entnommenen Beispiels verdeutlicht. Es handelt sich um die Navacerrada Brücke in Villalba, die von Huarte \& Cie., S. A., nach ainem Entwurf von Carlos Fernández Casado gebaut wurde. In einer vorausgegangenen Nummer dieser Zeitschrift ist bereits ausführlich über das Projekt berichtet worden. 